Preoperative staging of advanced ovarian cancer: comparison between ultrasound, computed tomography (CT) and whole-
body MRI with diffusion-weighted sequence (WB-DWI/MRI)

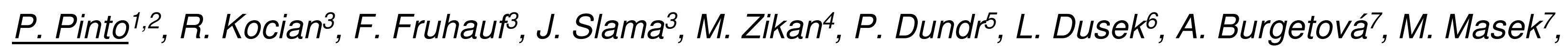 \\ D. Cibula ${ }^{3}, H$. Reina ${ }^{8}$, D. Fischerová ${ }^{3}$
}

\begin{abstract}
${ }^{1}$ Obstetrics and Gynecology, Maternidade Dr. Alfredo da Costa - Centro Hospitalar Universitário de Lisboa Central, EPE, Lisbon, Portugal, ${ }^{2}$ First Faculty of Medicine and General University Hospital, Charles University, ${ }^{3}$ Gynecologic Oncology Center, Department of Obstetrics and Gynecology, First Faculty of Medicine, Charles University in Prague and General University Hospital in Prague, Czech Republic, ${ }^{4}$ Obstetrics and Gynecology, Bulovka Hospital, First Faculty of Medicine, Charles University, ${ }^{5}$ Pathology, First Faculty of Medicine, Charles University in Prague and General University Hospital in Prague, Czech Republic, Prague, ${ }^{6}$ Institute of Biostatistics and Analysis, Masaryk University, Brno, ${ }^{7}$ Radiology, First Faculty of Medicine, Charles University in Prague and General University Hospital in Prague, Czech Republic, Prague, Czech Republic, ${ }^{8}$ Obstetrics and
\end{abstract} Gynecology, University Hospital Basel, Basel, Switzerland

Introduction / Background: To compare the diagnostic accuracy of ultrasound (US), CT and WB-DWI/MRI in preoperative staging and assessment of optimal operability in patients with ovarian cancer.

Methodology: Patients planned for ovarian cancer surgery were enrolled. They underwent preoperative work-up with US, CT and WB-DWI/MRI, following evaluation form. Findings were compared to the reference standard (intraoperative and histopathological evaluation forms).

Results: From 67 patients enrolled, 51 (76\%) had advanced and $16(24 \%)$ early stage ovarian cancer. Ultrasound showed the best results in the detection of pelvic carcinomatosis, in contrast with MRI and CT (AUC 0.94, 0.91, and 0.82, respectively), and in the evaluation of the depth of rectosigmoid infiltration (AUC 0.96, 0.81 , and 0.85 ). In the upper abdomen, all three methods showed comparable results in the detection of liver involvement (AUC $0.78,0.79$ and 0.76 for US, CT and WB-DWI/MRI), while US had the lowest AUC in the assessment of diaphragm (AUC 0.73, 0.84, 0.81). In the middle abdomen, ultrasound reached the highest AUC in the assessment of greater omentum and anterior wall (AUC of 0.87 and 0.76 ), in comparison with MRI (AUC 0.85 and 0.74 ), followed by CT (AUC 0.80 and 0.66 ). Ultrasound, MRI and CT showed comparable results in the assessment of bowel surface involvement (AUC 0.70, 0.69, 0.71), and ultrasound and MRI were better than CT (AUC 0.64, 0.71 and 0.76) in mesenterial involvement detection. In the assessment of retroperitoneal LNs, ultrasound and $\mathrm{MRI}$ were comparable (AUC 0.83), followed by CT (AUC of 0.76). All three modalities showed similar AUC in the prediction of optimal operability (AUC of 0.79 for US and CT and 0.80 for MRI).

Conclusion: This is the first prospective study documenting the potential role of ultrasound in ovarian cancer staging, compared to the method of choice (CT) and a novel technique (WB$\mathrm{DWI} / \mathrm{MRI})$.

Disclosure: This work was supported by a grant from the Czech Research Council (AZV NV19-03-00552).

\section{Mean age}

Advanced stage

Tubal cancer

High grade serous ca

Mean CA 125

Surgery

- Diagnostic laparoscopy $\quad 16 \%(11 / 67)$

- Primary debulking surgery $72 \%$ (48/67) - Combined LPS+LPT $\quad 12 \%(8 / 67)$

Macroscopic residuum (R2) 30\% (20/67)

\begin{tabular}{|c|c|}
\hline $\begin{array}{l}\text { Potential eligible participants }(n=81) \\
\text { Malignant adnexal mass or peritoneal } \\
\text { cancer on ultrasound } \\
\text { Suitable for surgical staging } \\
18>\text { Age }<80 \\
\text { ECOG PS }<3\end{array}$ & $\begin{array}{l}\text { Excluded }(n=6) \\
\text { Contraindications to } \mathrm{CT} \text { or MRI } \\
\text { Consent not given by the patient } \\
\text { Tru-cut biopsy proven secondary }\end{array}$ \\
\hline Eligible participants $(n=75)$ & \\
\hline & $\frac{\text { Excluded }(n=2)}{\text { No index test }}$ \\
\hline $\begin{array}{l}\text { US +CT + WB-DWIIMRI } \\
\text { ISAAC protocol }\end{array}$ & $\begin{array}{l}\text { Excluded }(n=4) \\
\text { No reference standard later than }\end{array}$ \\
\hline $\begin{array}{l}\text { Reference standard ( } \mathrm{n}=69 \text { ): } \\
\text { Surgery (DOL or laparotomy) } \\
\text { ISAAC protocol }\end{array}$ & $\frac{\text { Excluded }(n=2)}{\text { No compliance with ISAAC }}$ \\
\hline Final diagnosis $(n=67)$ & $\begin{array}{l}\text { protocol - no surgical form (1) } \\
\text { Secondary cancer (1) }\end{array}$ \\
\hline
\end{tabular}

Sensitivity of imaging in specific areas

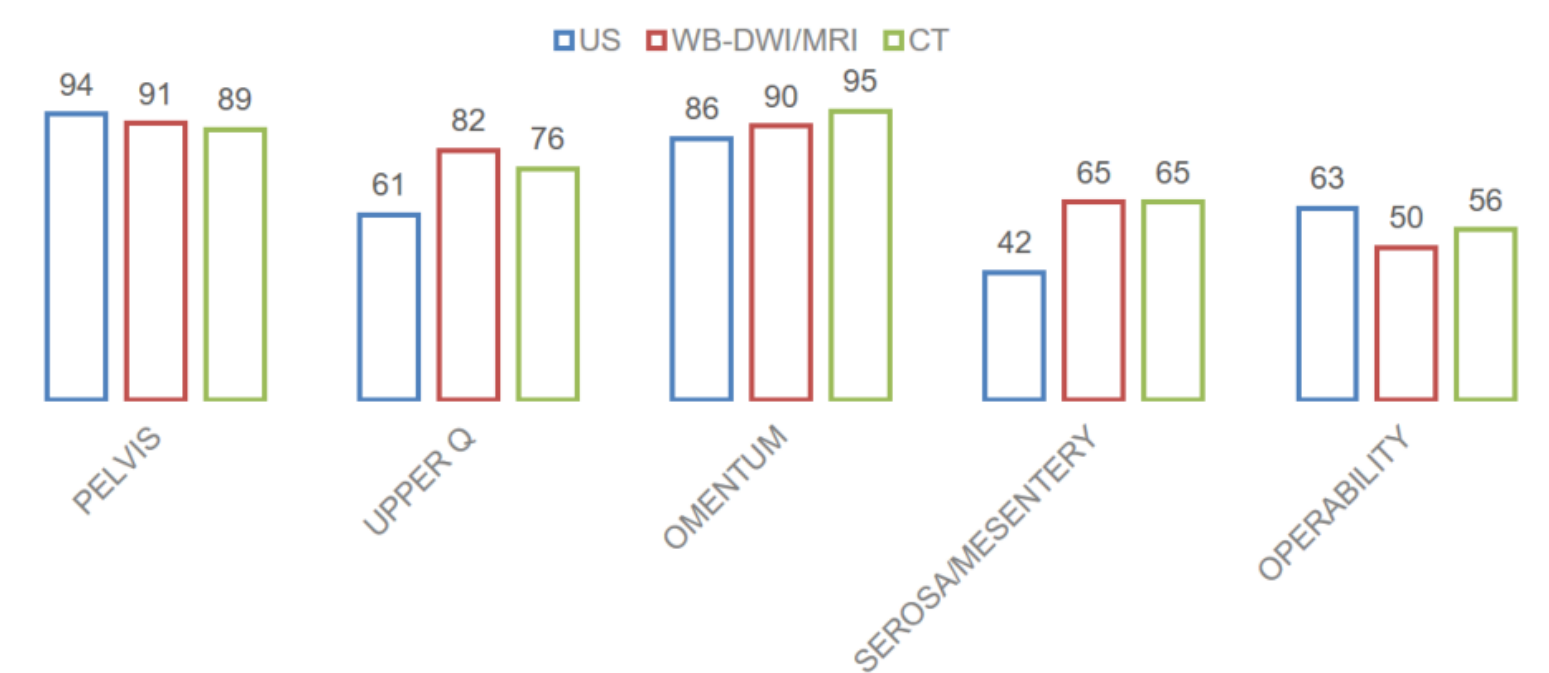

Specificity of imaging in specific areas aUS aWB-DWIMRI $\mathrm{QCT}$

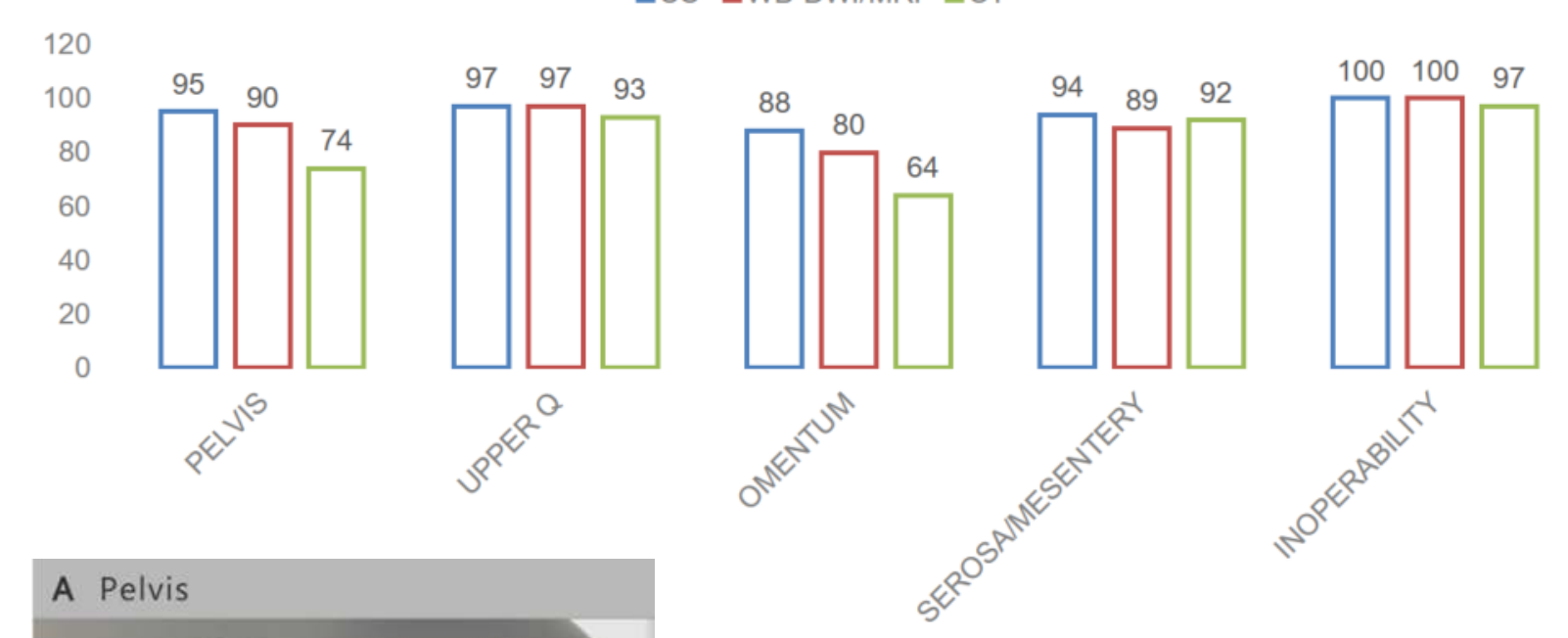

Overall peritoneal carcinomatosis assessment aUS aWB-MRI/DWI aCT

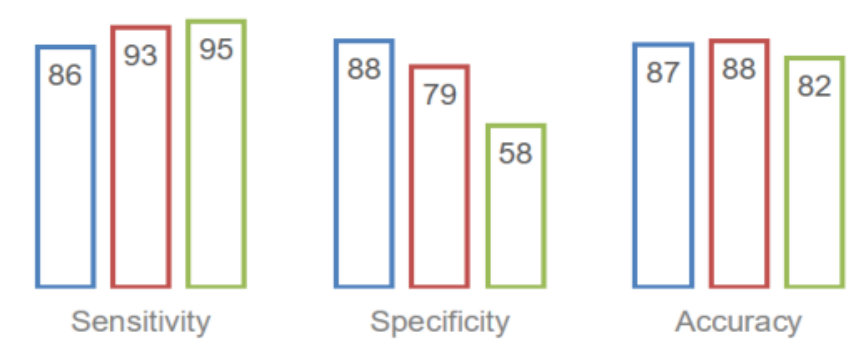

Retroperitoneal lymph nodes aUS aWB-DWI/MRI aCT
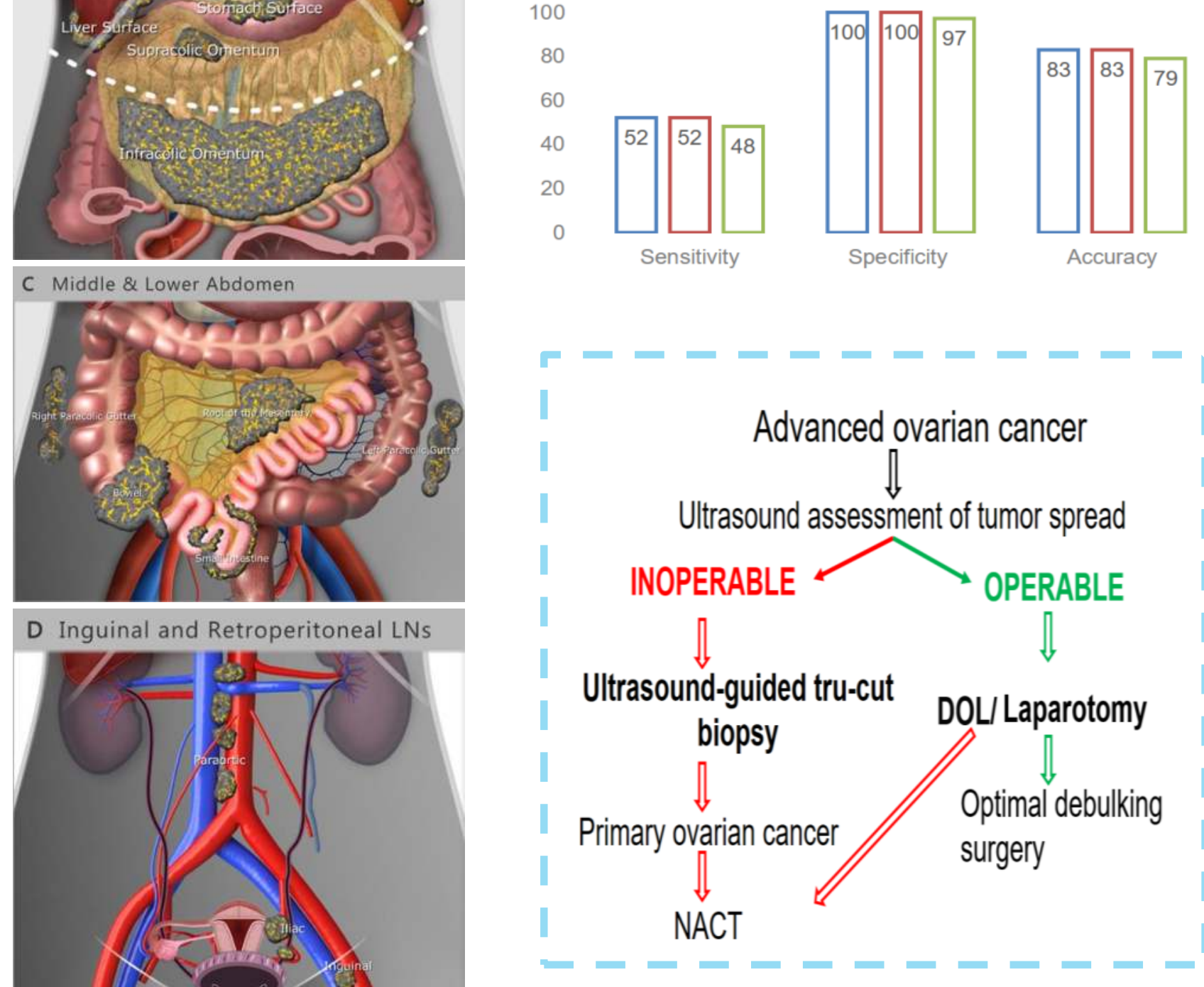